OPEN ACCESS

Edited by:

Fabrice Cognasse,

Groupe Sur L'immunité Des

Muqueuses Et Agents Pathogènes

(GIMAP), France

Reviewed by:

Anne L. Astier,

INSERM U1043 Centre de

Physiopathologie de Toulouse

Purpan, France

Lars Nitschke,

University of Erlangen

Nuremberg, Germany

*Correspondence:

Hongbin Yuan

hcf@immunol.org

Chaofeng Han

jfizcyy@aliyun.com

Yan Luo

ly11087@rih.com.cn

tThese authors have contributed equally to this work

Specialty section:

This article was submitted to Inflammation,

a section of the journa

Frontiers in Immunology

Received: 24 June 2019

Accepted: 17 October 2019

Published: 07 November 2019

Citation:

LiW, Li Y, Qin K, Du B, Li T, Yuan H, Han $C$ and Luo Y (2019) Siglec-G

Deficiency Ameliorates

Hyper-Inflammation and Immune Collapse in Sepsis via Regulating Src

Activation. Front. Immunol. 10:2575.

doi: 10.3389/fimmu.2019.02575

\section{Siglec-G Deficiency Ameliorates Hyper-Inflammation and Immune Collapse in Sepsis via Regulating Src Activation}

\author{
Wenqian $\mathrm{Li}^{1+}$, Yinjiao $\mathrm{Li}^{2+}$, Kewei Qin ${ }^{3+}$, Boxiang $\mathrm{Du}{ }^{4+}$, Tianliang $\mathrm{Li}^{5}$, Hongbin Yuan ${ }^{1 *}$, \\ Chaofeng Han $^{5 *}$ and Yan Luo ${ }^{2 *}$
}

${ }^{1}$ Department of Anesthesiology, Changzheng Hospital, Second Military Medical University, Shanghai, China, ${ }^{2}$ Department of Anesthesiology, Ruijin Hospital, Shanghai Jiaotong University School of Medicine, Shanghai, China, ${ }^{3}$ Central Laboratory, The Sixth Medical Center of Chinese PLA General Hospital, Beijing, China, ${ }^{4}$ The Second Affiliated Hospital of Nantong University, Nantong, China, ${ }^{5}$ National Key Laboratory of Medical Immunology, Institute of Immunology, Second Military Medical

University, Shanghai, China

Hyper-inflammation during acute phase and sequential hypo-inflammation during immunosuppressive phase in macrophages/monocytes lead to multiorgan failure syndrome and immune collapse of sepsis, in which toll-like receptor (TLR)-triggered inflammatory responses play a major role. Here, we reported that Siglecg deficiency attenuated TLR4-triggered pro-inflammatory cytokine production and increased antiinflammatory cytokine [interleukin-10 [IL-10]] production in vivo and in vitro at both acute and immunosuppressive phases. Siglecg deficiency also protected mice from lipopolysaccharide (LPS)-induced sepsis with less inflammation in the lung and less tissue destruction in the spleen. Siglec-G inhibited proto-oncogene tyrosine-protein kinase Src (Src) activation via recruiting and activating tyrosine phosphatase Src homology region 2 domain-containing phosphatase-1 (SHP1) through immunoreceptor tyrosine-based inhibitory motif (ITIM) domain. Src could inhibit TLR4-induced inflammatory cytokines and promote anti-inflammatory cytokine IL-10. Mechanical investigation showed that Src could interact with and phosphorylate STAT3. Src could also promote HIF1 $\alpha$ degradation through activating GSK3 $\beta$. Our study reveals that Siglec-G orchestrates TLR-induced inflammation, which outlines that blocking Siglec-G or activating Src may be a promising strategy for both acute and chronic inflammatory diseases.

Keywords: Siglec-G, Src homology region 2 domain-containing phosphatase-1, Src, sepsis, pro-inflammatory cytokines, anti-inflammatory cytokine

\section{INTRODUCTION}

Sepsis is a systemic inflammatory syndrome induced by infection, caused by the lack of normal immune homeostatic functions and excessive production of pro-inflammatory cytokines, which leads to multiorgan failure and immune collapse (1-7). Toll-like receptor (TLR) signaling and inflammatory responses are critical in the pathology of sepsis. By recognizing pathogen-associated molecular patterns (PAMPs), TLRs initiate innate immune responses by activating signaling pathways that depend on the adaptor myeloid differentiation primary response 88 (MyD88) or TIR-domain-containing adapter-inducing interferon- $\beta$ (TRIF) and consequently induce the 
production of pro-inflammatory cytokines and type I interferon (IFN) (8-10). The positive and negative regulation of TLR signaling has been under intensive research $(9,10)$. Diverse signaling molecules have been identified to be essential for full activation of TLR responses $(9,10)$. However, negative regulators of TLR such as Src (11) can prevent over-activation of TLR signaling, which may result in inflammatory disorders or autoimmune diseases $(12,13)$. We previously found that CD11bactivated Src signaling could inhibit inflammatory cytokine in sepsis (11) and could promote anti-inflammatory cytokine IL10 in mice model of colitis (14). Whether, there is a signal pathway regulating both inflammatory and anti-inflammatory cytokines in acute inflammation remains unknown $(9,15)$. Searching the balance point of pattern recognition receptor (PRR) signal transduction to produce appropriate production of anti-inflammatory and pro-inflammatory cytokines is a key question for innate responses and a promising drug target $(9,15)$.

Siglecs are divided into two groups on the basis of their molecular structure. The first group includes Siglec-1, Siglec2, Siglec-4, and Siglec-15, which are structurally conserved between rodents, humans, and other vertebrates (16-19). The second group includes Siglec-3/CD33 and CD33-related Siglecs, which are less structurally conserved between human and other vertebrates but highly homologous to CD33 in their extracellular domains. Most Siglecs bind sialic acid ligands either in-cis (on the same cell) or in-trans (on a neighboring cell or on a microbe) and provide inhibitory signals to immune cells. Siglecs play an important role in cell signal transduction, pathogen recognition, phagocytosis, and treatment of tumors. Siglec15 could induce immunosuppressive tumor microenvironment via immunoreceptor tyrosine-based activation motif (ITAM) domain of DAP12 (20). Our lab first cloned Siglec-10 from human dendritic cells (DCs) (21), which is a member of the CD33-related Siglec family in the mouse. Recent studies demonstrated that Siglec-G had a board negative modulation on B cells (17). Siglec-G also inhibited DC cross-presentation by impairing major histocompatibility complex (MHC)-I peptide complex formation (22). In innate immunity, inflammatory responses to host danger-associated molecular patterns (DAMPs) are repressed by the interaction of CD24 and Siglec-G (23, 24). We previously reported that Siglec-G negatively regulated RNA virus-induced type I IFN production by promoting cCbl-mediated ubiquitination and degradation of retinoic acidinducible gene 1 (RIG-I) via SHP2 (25). Most Siglecs have ITIM or ITIM-like motifs in intracellular domains. Upon ligand recognition, Siglecs recruit tyrosine phosphatases such as SHP1 or SHP2 to regulate signal transduction through ITIM or ITIMlike domain $(22,25,26)$. Furthermore, Siglec-G deficiency decreased infiltration of inflammatory cells and inflammation in fat tissue (27), which is consistent with the negative function of ITAM in inflammation $(12,28)$. However, the role of ITIMcontaining Siglec-G and the function of SHP1/2 in acute inflammation remain unclear.

In this study, we observed that Siglecg deficiency protected mice from over-activation of acute inflammatory responses and death in TLR-triggered sepsis by attenuating TLRtriggered pro-inflammatory cytokine production and increasing anti-inflammatory cytokine IL-10 production. We further demonstrated that Siglec-G decreased Src activation through SHP1. Our results showed that Siglec-G-induced Src signaling could be a promising drug target to regulate immune homeostasis of pro-inflammation and anti-inflammation.

\section{RESULTS}

\section{Siglec-G Orchestrates Toll-Like Receptor-Triggered Inflammatory and Anti-inflammatory Cytokine Productions in vivo}

We first found that the baseline expression of Siglec-G was not the highest, but its expression was upregulated significantly upon lipopolysaccharide (LPS) stimulation (Supplementary Figure 1). To investigate the role of Siglec-G in the inflammatory responses of sepsis, we challenged Siglecg-deficient $\left(\right.$ Siglecg $^{-/-}$) and control $\left(\right.$ Siglecg $\left.{ }^{+/-}\right)$mice with a non-lethal dose of LPS for indicated time, a sepsis model we previously reported (29). In response to the first LPS challenge, Siglecg-/- mice produced less interleukin-6 (IL-6) and tumor necrosis factor- $\alpha$ (TNF- $\alpha$ ) than did Siglecg ${ }^{+/-}$mice (Figure 1A). However, Siglecg ${ }^{-/-}$mice produced more anti-inflammatory cytokine IL-10 than did Siglecg ${ }^{+/-}$mice. Twenty-four hours after the first LPS challenge, the second LPS challenge induced significant lower production of IL- 6 and TNF- $\alpha$, which implicated an immunosuppressive status. Siglecg deficiency also significantly decreased the second LPS-induced pro-inflammatory cytokine (IL-6 and TNF- $\alpha$ ) productions, whereas it increased the anti-inflammatory cytokine IL-10 production compared with that in the control mice. We observed more severe infiltration of inflammatory cells in the lungs of Siglecg ${ }^{+/-}$mice than that in the lungs of Siglecg ${ }^{-/-}$ mice at both acute and immunosuppressive phases (Figure 1B). Accordingly, there was less activation of NF- $\kappa \mathrm{B}$ (phosphorylation of 665 subunit) and tissue destruction in the spleen of Siglecg ${ }^{-/-}$ mice than in Siglecg ${ }^{+/-}$mice. Upon lethal LPS-dose challenge, $60 \%$ of Siglecg ${ }^{+/-}$mice remained alive for $16 \mathrm{~h}$, but only $20 \%$ of them ultimately survived, whereas $80 \%$ of Siglecg $^{-/-}$mice ultimately survived (Figure 1C), which indicated that Siglecg-/mice were significantly more resistant to LPS-induced sepsis than were control mice. These results suggest that Siglecg deficiency protects mice from sepsis in both acute and immunosuppressive phases by orchestrating TLR-triggered inflammatory responses by inhibiting NF- $\mathrm{B}$ activation.

\section{Siglec-G Orchestrates Toll-Like Receptor-Triggered Inflammatory and Anti-inflammatory Cytokine Productions in Macrophages}

The above data indicated that Siglec-G orchestrated TLRtriggered inflammatory and anti-inflammatory cytokine productions in vivo. To further confirm the function of Siglec-G in vitro, LPS-induced cytokine production in mouse peritoneal macrophages was tested using ELISA. In response to LPS administration, Siglecg ${ }^{-/-}$peritoneal macrophages produced less pro-inflammatory cytokines (IL- 6 and TNF- $\alpha$ ) but more 

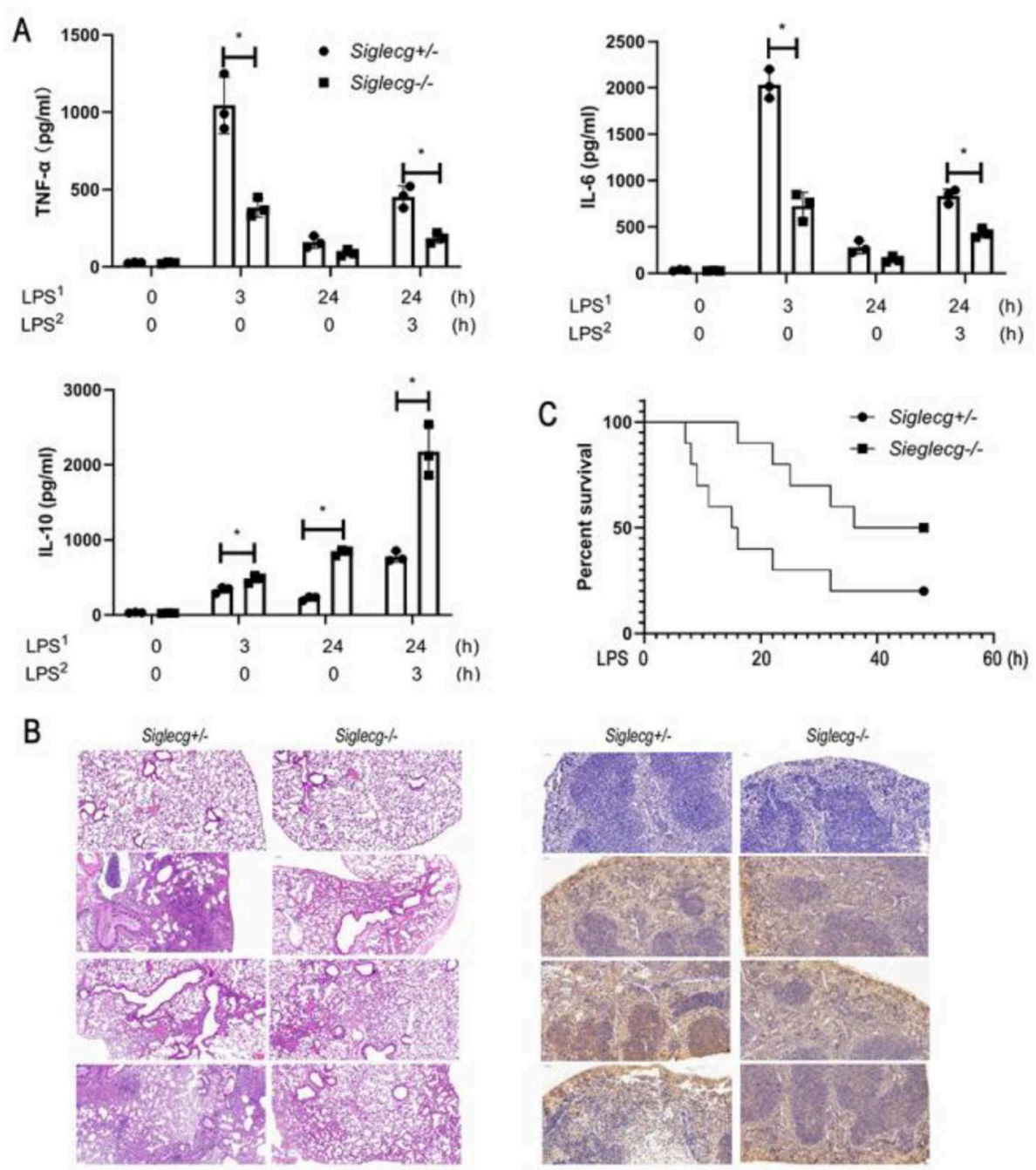

FIGURE 1 | Siglecg-deficient mice are more resistant to LPS-induced sepsis. (A) Enzyme-linked immunosorbent assay (ELISA) of TNF- $\alpha$, IL-6, and IL-10 in serum

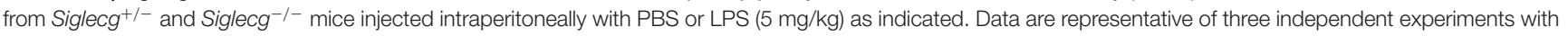
similar results and presented as means $\pm S D$. ${ }^{*} P<0.01$. (B) Hematoxylin and eosin staining of the lungs (left panel); the immunostaining of phosphor-p65 (middle panel) in the spleen from Siglecg ${ }^{+/-}$and Siglec ${ }^{-/-}$mice after intraperitoneal injection of PBS or LPS as indicated. Original magnification: $\times 10$ for the lungs and $\times 20$ for the spleen. Data are representative of three independent experiments with similar results. (C) Survival of Siglecg ${ }^{+/-}$and Siglecg ${ }^{-/-}$mice $(n=10$ per genotype), monitored every hour after challenge with lethal dose of LPS (15 mg/kg). $P<0.01$ (Wilcoxon test). LPS, lipopolysaccharide; IL, interleukin; TNF- $\alpha$, tumor necrosis factor- $\alpha$; PBS, phosphate-buffered saline.

IL-10 than did Siglecg ${ }^{+/-}$(Figure 2A, Supplementary Figure 2) and Siglecg $^{+/+}$(Supplementary Figure 2) macrophages at both acute and immunosuppressive phases. The mRNA expression of pro-inflammatory cytokines also decreased, and the mRNA expression of IL-10 increased in $\left(\right.$ Siglecg $^{-/-}$) macrophages (Supplementary Figure 3). Then we further investigated whether overexpression of Siglec-G could increase pro-inflammatory cytokines production in RAW264.7 cells. Overexpression of Siglec-G-HA significantly increased LPS-induced IL- 6 and TNF- $\alpha$ production and decreased IL-10 production than in control cells at both acute and immunosuppressive phases (Figure 2B). The knockout and overexpression efficiency was verified in the next part
(Figures 3A,C). These data further confirmed that Siglec-G could promote TLR-trigged pro-inflammatory cytokine production and inhibit anti-inflammatory cytokine IL-10 production in vitro.

\section{Siglec-G Orchestrates NF-кB and Extracellular Signal-Regulated Kinase Activation}

To further explore how Siglec-G promotes TLR-induced proinflammatory cytokine production, the downstream signal pathway was tested. The expression of Siglec-G in macrophages and knockout efficiency were first verified. We found that the 

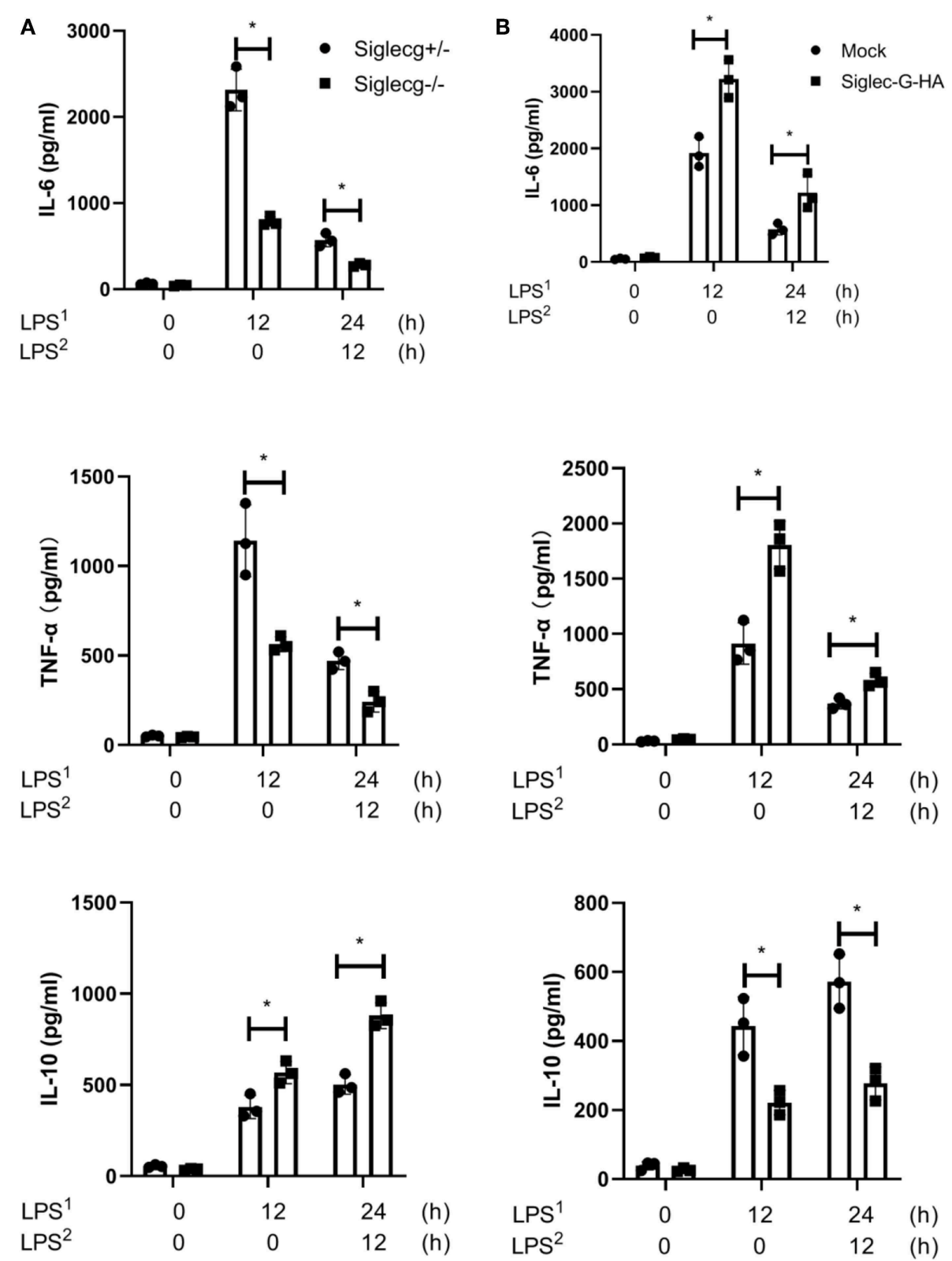

(h)

(h)

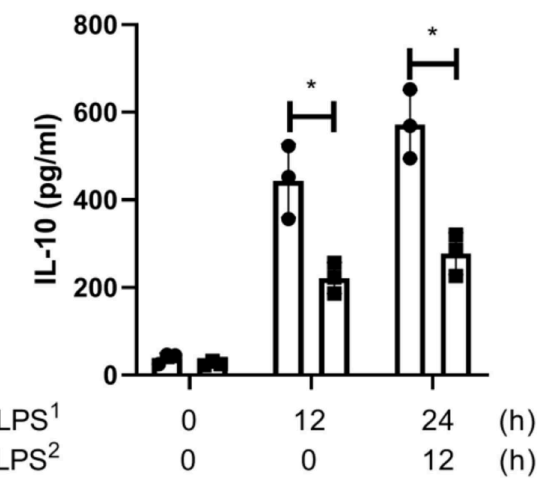

FIGURE 2 | Siglec-G orchestrates TLR-triggered pro-inflammatory and anti-inflammatory cytokines production in macrophage. (A) ELISA of IL-6, TNF- $\alpha$, and IL-10 in supernatant from Siglecg ${ }^{+/-}$and Siglecg ${ }^{-/-}$peritoneal macrophages $\left(6 \times 10^{5}\right)$ that were stimulated with first round of LPS (100 ng/ml) as indicated or were changed with then fresh medium for the second round of LPS for $12 \mathrm{~h}$. (B) ELISA of IL-6, TNF- $\alpha$, and IL-10 in supernatant from Siglec-G-overexpressing RAW264.7

macrophages $\left(8 \times 10^{5}\right)$ stimulated with LPS as $(\mathbf{A})$. Data are representative of three independent experiments with similar results and presented as means \pm SD. ${ }^{*}<$ 0.01. TLR, toll-like receptor; IL, interleukin; TNF- $\alpha$, tumor necrosis factor- $\alpha$; LPS, lipopolysaccharide.

activation of NF-кB was impaired in Siglecg ${ }^{-/-}$macrophages compared with that in Siglecg ${ }^{+/-}$macrophages (Figure 3A), which was evidenced by decreased phosphorylated IKK $\alpha / \beta$ and p65 levels. However, the activation of extracellular signalregulated kinase (ERK), which is critical for TLR-induced IL-10 production (30), was increased in Siglecg ${ }^{-/-}$macrophages. The nuclear translocation of NF-кB transcriptor p65 was decreased, and the ERK-induced transcriptor AP-1 (c-jun) was increased in Siglecg ${ }^{-/-}$macrophages than in Siglecg ${ }^{+/-}$macrophages (Figure 3B). The Siglec-G was overexpressed efficiently in RAW264.7 cells. We also tested whether Siglec-G overexpression could orchestrate the activation of NF-кB and ERK in RAW264.7 cells. In accordance with results of Siglecg-deficient macrophages, the activation of NF- $\kappa$ B was also increased in Siglec-G-HA overexpressed RAW264.7 cells (Figure 3C). And Siglec-G-HA overexpression decreased the activation of ERK in RAW264.7 
A

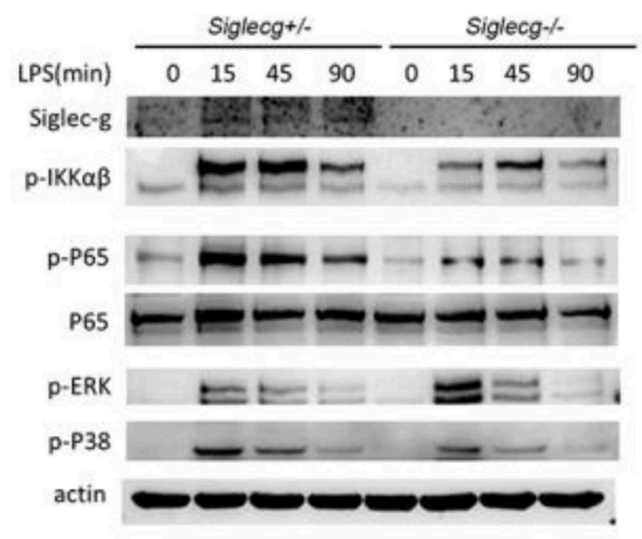

B

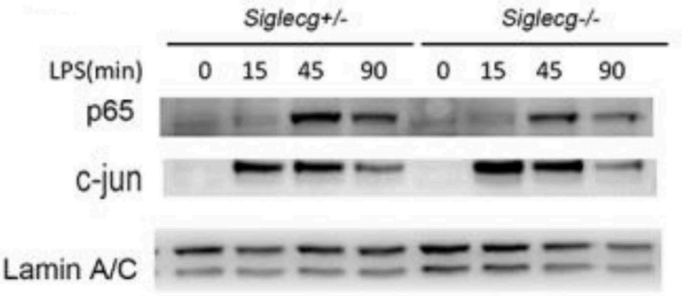

C

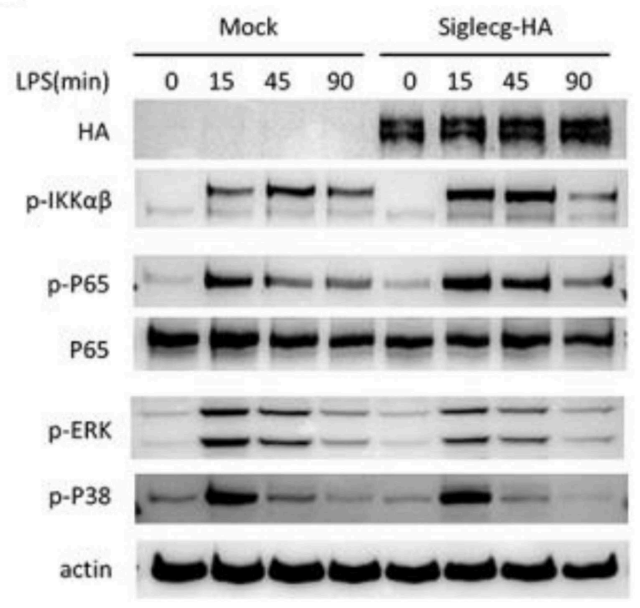

D

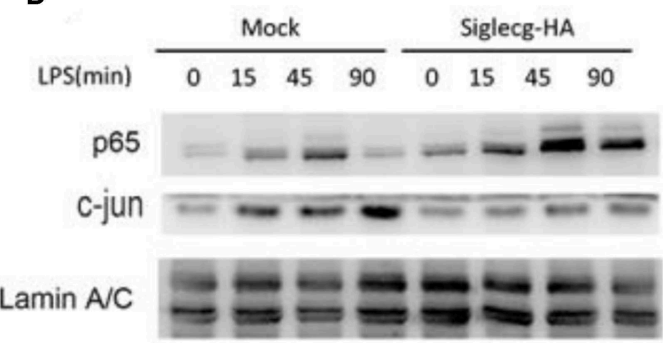

FIGURE 3 | Siglec-G orchestrates NF-kB and ERK activation. (A,B) Immunoblotting of cell lysates (A) or nuclear extract (B) from Siglecg ${ }^{+/-}$and Siglecg ${ }^{-/-} 2 \times 10^{6}$ peritoneal macrophages stimulated with LPS $(100 \mathrm{ng} / \mathrm{ml})$ for the indicated time with indicated antibodies. (C,D) Immunoblotting of cell lysates (C) or nuclear extract (D) from Siglec-G overexpressing RAW264.7 cells $\left(3 \times 10^{6}\right)$ stimulated with LPS $(100 \mathrm{ng} / \mathrm{ml})$ for the indicated time with indicated antibodies. Data are representative of three independent experiments with similar results. ERK, extracellular signal-regulated kinase; LPS, lipopolysaccharide.

cells. Moreover, similar results were confirmed by the nuclear translocation of p65 and AP-1 (c-jun) in Siglec-G overexpressed cells (Figure 3D). The above data suggested that Siglec-G could orchestrate TLR-induced NF- $\kappa \mathrm{B}$ and ERK activation.

\section{Siglec-G Orchestrates Toll-Like Receptor Signaling Through Src}

We previously found that CD11b-induced Src activation was involved in TLR signaling $(8,9)$. The activation of Src was increased in Siglecg ${ }^{-/}$peritoneal macrophages upon LPS stimulation (Figure 4A). And Siglec-G overexpression impaired Src activation (Figure 4B). Pretreatment of wild-type (WT) peritoneal macrophages and Siglecg-/- peritoneal macrophages with Src inhibitor PP1 increased pro-inflammatory cytokine (IL-6 and TNF- $\alpha$ ) production (Figure 4C) at both the protein and mRNA levels (Supplementary Figure 4). Accordingly, PP1 also decreased IL-10 expression. PP1 increased proinflammatory cytokines production more efficiently in Siglecgdeficient macrophages (Figure 4C), in which there was more strength of Src activation (Figure 4A). To further confirm the role of Src in TLR signaling, we pretreated Siglec-G overexpressed or control RAW264.7 cells with PP1. PP1 also increased IL-6 and
TNF- $\alpha$ production and decreased IL-10 production in control RAW264.7 cells (Figure 4D), but not in Siglec-G overexpressed cells, in which the activation of Src was inhibited (Figure 4B). The above data indicated that Siglec-G regulated NF- $\kappa$ B and ERK activation through Src.

\section{Siglec-G Inhibits Src Activation via Src Homology Region 2 Domain-Containing Phosphatase-1}

We further investigated how Siglec-G inhibited Src activation upon LPS stimulation. As we previously noticed a positive function of the tyrosine phosphatase SHP1 in innate immunity $(28,31)$, we investigated whether the positive function of Siglec-G was dependent on SHP1. Overexpression and co-IP experiments in HEK293T cells indicated that Src interacted with SHP1 (Figure 5A). Furthermore, we found that SHP1 could dephosphorylate overexpressed Src in a dose-dependent manner (Figure 5B). Overexpression of Siglec-G, SHP1, and Src in HEK293T cells indicated that Siglec-G could promote dephosphorylation of Src, which was further enhanced by SHP1 (Figure 5C). SHP1 could also interact with Siglec-G and Src upon LPS stimulation in macrophages (Figure 5D). Siglec-G contains 


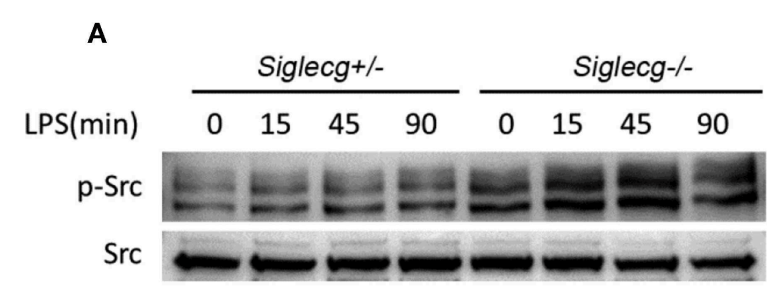

B
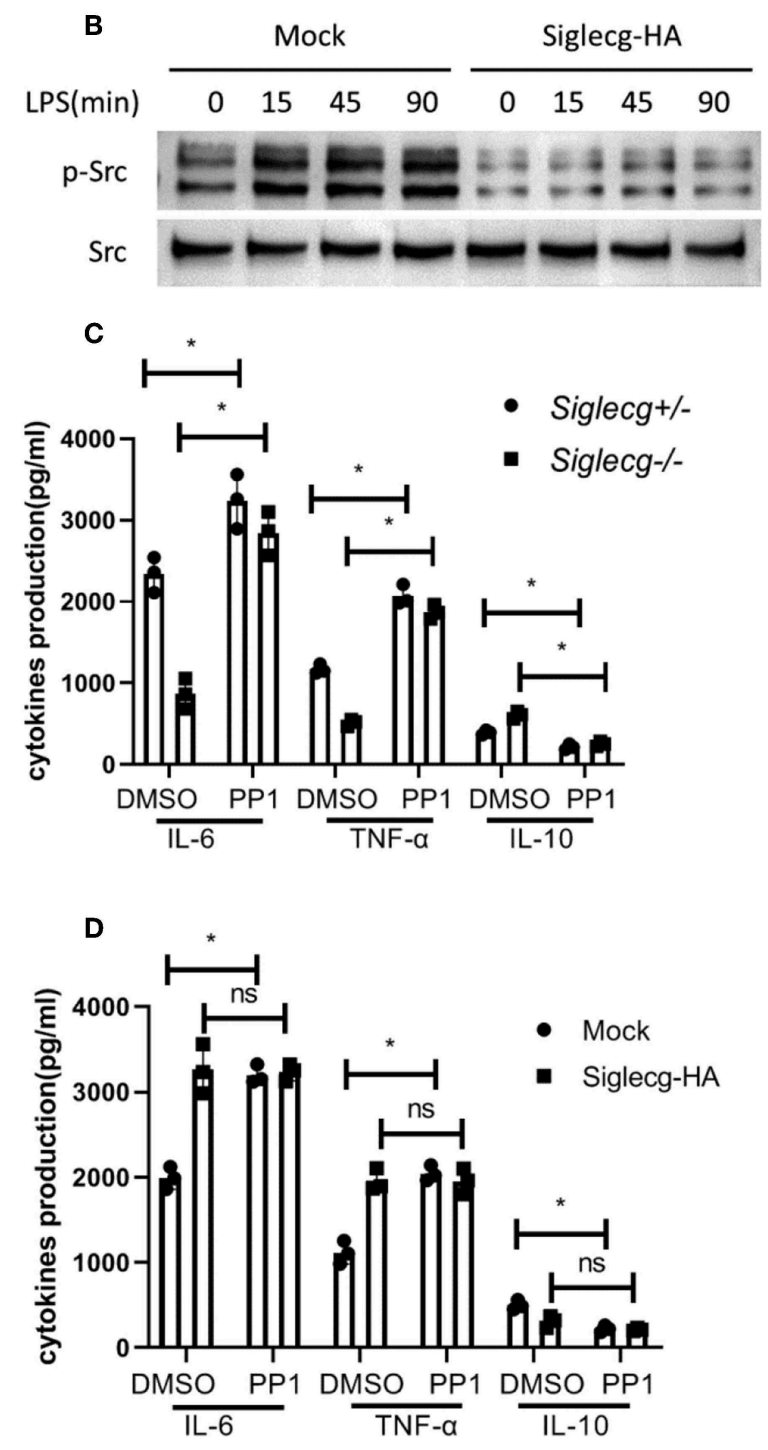

FIGURE 4 | Siglec-G inhibits Src activation. (A,B) Immunoblotting of cell lysates from $2 \times 10^{6}$ Siglecg $^{+/-}$and Siglecg ${ }^{-/-}$peritoneal macrophages (A) or $3 \times 10^{6}$ Siglec-G overexpressing RAW264.7 cells (B) stimulated with LPS $(100 \mathrm{ng} / \mathrm{ml})$ for the indicated time with indicated antibodies. Data are representative of three independent experiments with similar results. (C,D) ELISA of IL-6, TNF- $\alpha$, and IL-10 in supernatant from Siglecg ${ }^{+/-}$and Siglecg-/- peritoneal macrophages (C) or Siglec-G overexpressing RAW264.7 macrophages (D) pretreated with PP1 $(5 \mu \mathrm{M})$ for $2 \mathrm{~h}$ and then stimulated with LPS $(100 \mathrm{ng} / \mathrm{ml})$ for $12 \mathrm{~h}$. Data are representative of three independent experiments with similar results and presented as means \pm SD (ns, no significant differences; * $P<0.01$ ). LPS, lipopolysaccharide; IL, interleukin; TNF- $\alpha$, tumor necrosis factor- $\alpha$. an intracellular tail with four tyrosine-based motifs, one or two belonging to the ITIM domain. The Siglec-G ITIM inactive mutant (Siglec-G-4YF) decreased the inhibitory function on Src activation compared with the full-length Siglec-G (WT), whereas the cytoplasmic domain-deleted Siglec-G (DEL) mutant totally lost the inhibitory function on Src activation (Figure 5E) in the overexpressed system. These results indicated that Siglec-G could decrease Src activation by recruiting SHP1.

\section{Siglec-G Orchestrates STAT3 and HIF1 $\alpha$ Activation via Src}

We investigated how Src orchestrated the inflammatory signaling activation. HIF $1 \alpha$ is important in LPS-induced inflammatory response (32). We found that Siglecg $^{-/-}$ macrophages exhibited lower HIF1 $\alpha$ protein expression than Siglecg $^{+/-}$macrophages upon LPS stimulation (Figure 6A). GSK3 $\beta$ could phosphorylate HIF1 $\alpha$ and promote its degradation in proteasome. Accordingly, the activation of GSK3 $\beta$ was increased in Siglecg ${ }^{-/-}$macrophages compared with Siglecg ${ }^{+/-}$ macrophages. Furthermore, the activation of STAT3 is critical for IL-10 production $(30,33)$. Its activation increased in Siglecg ${ }^{-/-}$ macrophages compared with Siglecg ${ }^{+/-}$macrophages upon LPS stimulation. We previously found that Src-activated SrcAkt-GSK3 signaling could activate AP-1 (14). Then we further investigated how Siglec-G-mediated Src signaling could increase AP- 1 and STAT3 activation and IL-10 production. The activation of inflammatory signaling of NF- $\mathrm{kB}$ and HIF $1 \alpha$ was decreased, while activation of anti-inflammatory signaling of AP-1, STAT3, and GSK3 $\beta$ was increased in RAW264.7 cells overexpressed constitutively active Src (CA-Src) (Figure 6B). Accordingly, RAW264.7 cells that overexpressed CA-Src also produced less IL-6, while they produced more IL-10 (Figure 6C). These data indicated that Src might promote HIF $1 \alpha$ degradation and STAT3 activation to orchestrate inflammatory responses.

\section{Src Is Involved in STAT3 and HIF1 $\alpha$ Activation}

Then we further investigated the role of Src in regulating STAT3 and HIF $1 \alpha$ activation. We co-overexpressed STAT3 with increased amounts of CA-Src in HEK293 cells (Figure 7A). Overexpressed CA-Src could interact with STAT3 and phosphorylated STAT3. CA-Src could increase the phosphorylation with STAT3 in a dose-dependent manner. When HIF1 $\alpha$ was co-overexpressed with increased amounts of CA-Src in HEK293 cells, CA-Src could also interact with GSK3 $\beta$ and HIF1 $\alpha$ (Figure 7B). CA-Src could also increase the phosphorylation of GSK $3 \beta$ and decrease the expression HIF1 $\alpha$ in a dose-dependent manner. The above results show that Src was involved in the inflammatory signaling by regulating STAT3 and HIF $1 \alpha$ activation.

\section{DISCUSSION}

Sepsis is defined as a life-threatening organ dysfunction with systemic mixed pro-inflammatory and anti-inflammatory responses to an infectious organism or severe tissue injury 

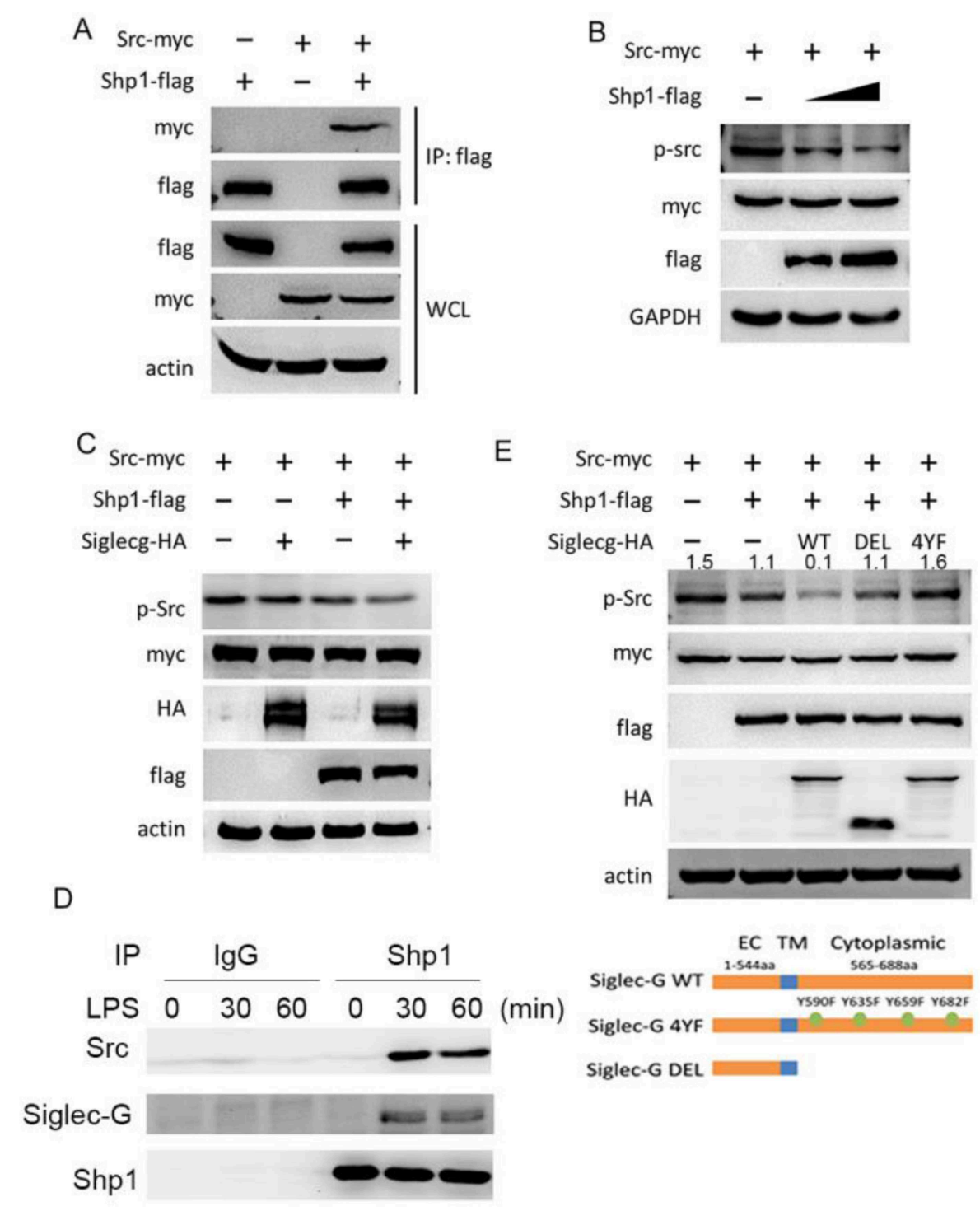

FIGURE 5 | Siglec-G inhibits of Src activation via SHP1. (A) Immunoblotting of immunoprecipitated production or cell lysates from HEK293T cells overexpressing indicated plasmids. (B,C) Immunoblotting of the cell lysates from HEK293T cells overexpressing indicated plasmids. (D) Immunoblotting of immunoprecipitated production from macrophages with indicated antibodies. (E) Immunoblotting of the cell lysates from HEK293T cells overexpressing indicated plasmids. Data are representative of three independent experiments with similar results. SHP1, Src homology region 2 domain-containing phosphatase-1.

$(1,6,7,15,34)$. Despite the failure of numerous clinical trials, anti-inflammatory treatment strategies in the early hours after the onset of sepsis are still a promising option (3). Indeed, clinical studies showed that subgroups of patients with sepsis might benefit from anti-inflammatory treatment strategies such as IL-1 receptor blockade or anti-TNF treatments $(6,7,34)$. Here, we found that Siglec-G deficiency orchestrated the proinflammatory cytokines and anti-inflammatory cytokine IL-10, which protected mice from LPS-induced sepsis. Interestingly, applying inhibition of Siglec-G downstream Src or activating Src could significantly orchestrate the pro-inflammatory cytokine and anti-inflammatory productions in macrophages. Although anti-inflammatory cytokines blocking therapy will cause protracted immunosuppressive phase in most patients $(5-7,15,34)$, our results indicated that blocking Siglec-G (activating $\mathrm{Src}$ ) in hyper-inflammation phase or activating Siglec-G (inhibiting Src) in hypo-inflammation phase might be beneficial for septic patients. Thus, a precise therapy for sepsis should firstly discriminate the immune status.

Siglec-G is an immunoglobulin-like lectin molecule that recognizes sialic acid and $\operatorname{CD} 24(17,26)$. By interacting with CD24, Siglec-G suppresses inflammatory responses to DAMP such as heat-shock proteins (Hsp70 and Hsp90) and high mobility group box 1 protein (HMGB1) and also regulating TLR activation $(23,24)$. Siglec-G/10 plays an important role in self-non-self discrimination of innate immunity. In 


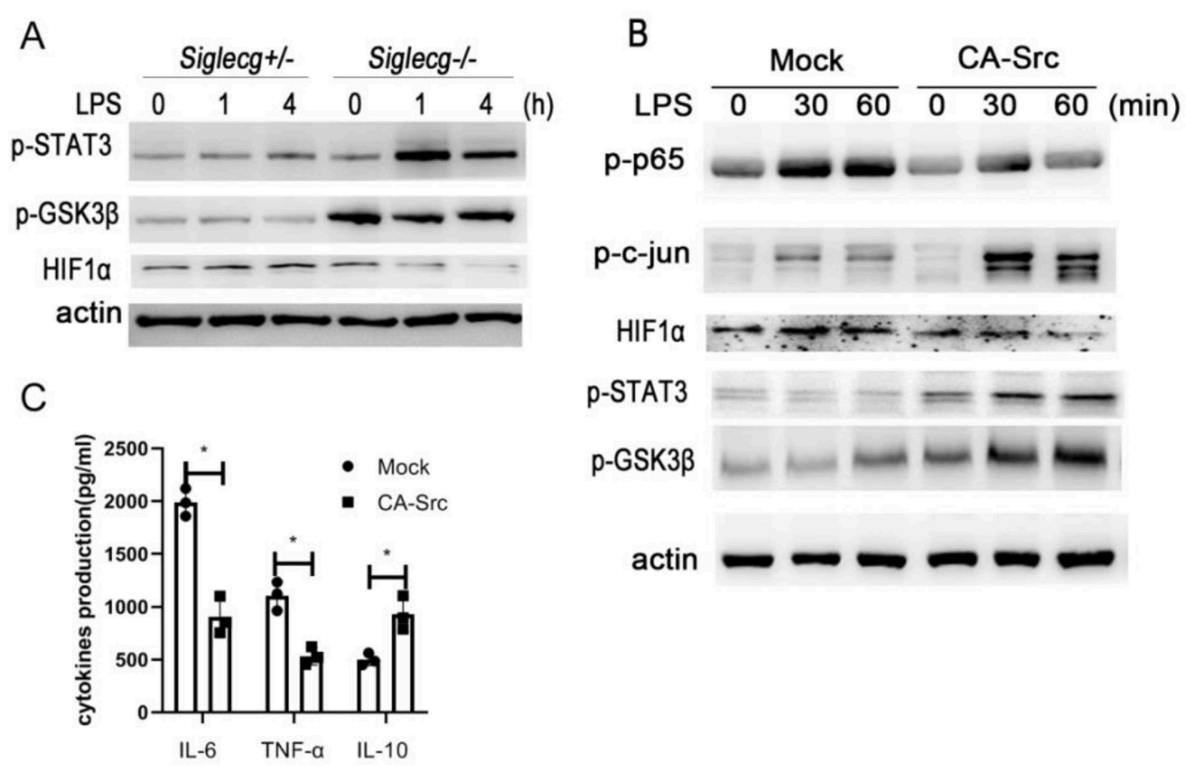

FIGURE 6 | Siglec-G orchestrates TLR4-induced STAT3 and HIF1 $\alpha$ activation via Src. (A,B) Immunoblotting of cell lysates from Siglecg ${ }^{+/-}$and Siglecg-/- peritoneal macrophages or RAW264.7 cells overexpressing CA-Src stimulated with LPS (100 ng/ml) for indicated time with indicated antibodies. (C) ELISA of IL-6, TNF- $\alpha$, and IL-10 in supernatant from constitutively active Src (CA-Src) overexpressing RAW264.7 cells stimulated with LPS (100 ng/ml) for $12 \mathrm{~h}$. Data are representative of three independent experiments with similar results as means \pm SD. ${ }^{\star} P<0.01$. TLR, toll-like receptor; LPS, lipopolysaccharide; IL, interleukin; TNF- $\alpha$, tumor necrosis factor- $\alpha$.

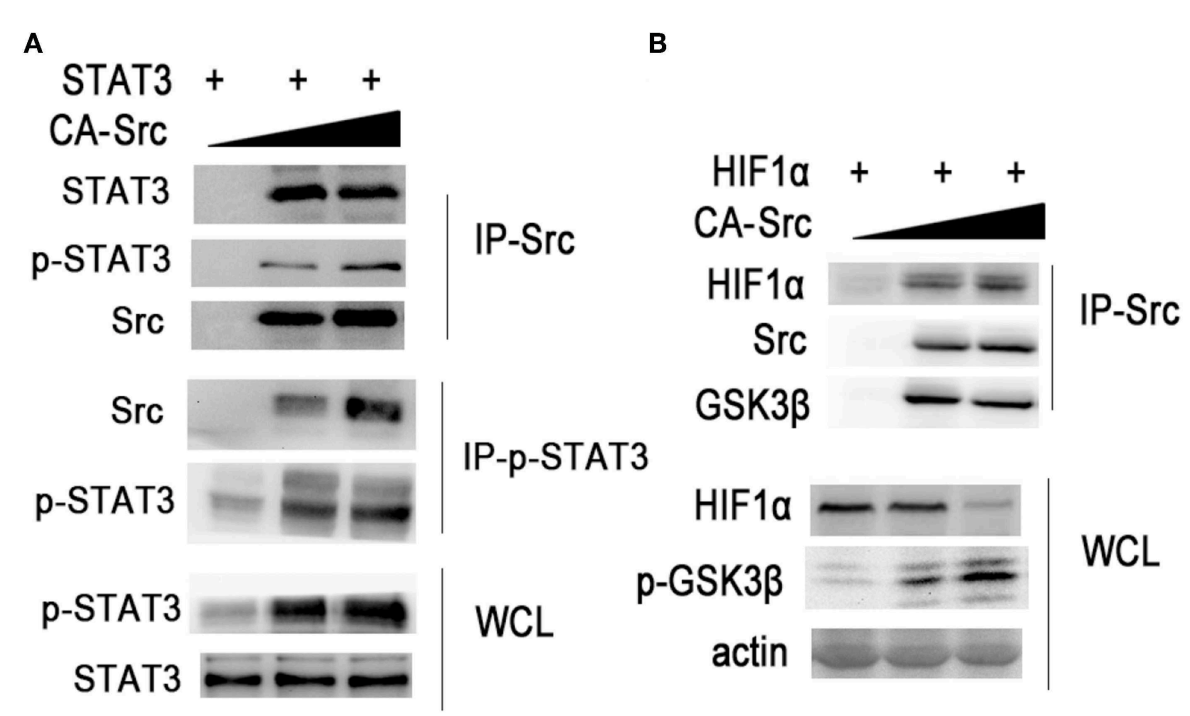

FIGURE 7 | Src regulates STAT3 and HIF1 $\alpha$ activation. (A,B) Immunoblotting of immunoprecipitated production or cell lysates from HEK293T cells overexpressing indicated plasmids with indicated antibodies. Data are representative of three independent experiments with similar results.

animals, sialic acid-bearing glycans, which are expressed on the cell surface as components of membrane glycoproteins or of a class of glycolipids called gangliosides, forms a key component of the glycocalyx and is critically involved in cell-cell interactions and cell signaling (17, 26). Diverse functions of inflammatory responses induced by DAMP or PAMP may result from different expression levels of Siglec$G$ and sialic acid-bearing glycans on the membrane, which leads to activation of SHP1 or SHP2, with varying degrees. Furthermore, the interaction of Siglec-G with sialic acid from membrane proteins (like CD24) or other proteins may be disrupted by sialidases such as neuraminidase. Neuraminidase was upregulated in our previous gene expression assay (25). Neuraminidase inhibitor is used as an antiviral drug, typically for influenza infection. Our unpublished data suggested that neuraminidase inhibitor might have a function similar to 
that of Siglec-G on TLR4-induced signaling and response, indicating that neuraminidase inhibitor might increase the following bacteria-induced inflammation. The function of the neuraminidase inhibitor and Siglecg deficiency effect on TLR signaling and responses indicated that Siglec-G was an important member of Siglecs on macrophages. Further research of neuraminidases on inflammation expressed from macrophages, other cells, or pathogens will provide a novel mechanism of innate immunity.

Our previous work showed that Siglec-G associated with c-Cbl caused degradation of RIG-I and reduced production of type I IFN in response to RNA virus infection in a CD24-independent manner (25). Here, we found that Siglec-G promoted TLR-triggered pro-inflammatory cytokines expression both in vivo and in vitro by regulating Src activation through SHP1. Siglecs can bind sialic acid-modified substrates through immunoglobulin-like receptors, which discriminate between self and non-self and regulate the function of cells in the innate and adaptive immune systems $(18,26)$. Siglec- $\mathrm{G}$ was reported to bind sialic acid on CD24 to downregulate TLR4 signaling in-cis manner (on the same cell) $(23,24)$. Siglec-G could also participate in autoimmune disease by cooperating with inhibitory receptor Fc $\gamma$ RIIb (35). However, Fc $\gamma$ RIIb has also a negative function on TLR4 signaling via the ITAM domain $(12,13)$. Recently, tumor cells that expressed CD24 will inhibit phagocytosis by macrophages through binding Siglec-G in-trans manner (36), which also indicates that different substrate recognition patterns of Siglec-G. We speculated that the interaction with different sialic acid-modified substrates and different expression statuses of Siglec-G upon LPS stimulation might induce different signaling pathways. Different gene backgrounds of control mice, knockout efficiency, and experimental model will also influence the function of Siglec-G on inflammation $(23,24)$. We focused on function of Siglec-G on the both acute and immunosuppressive phases of LPS-induced sepsis model, which is much different from the cecal ligation and puncture (CLP)-induced sepsis model. The rigid control mice with the same gene background (Siglecg $^{+/-}$vs. Siglecg ${ }^{-/-}$), which is more suitable to illustrate the function of Siglec-G. Siglecs are also endocytic receptors that constitutively cycle between the cell surface and intracellular endosome ligand-bearing cargo into the cell, in which the molecular basis for sialic acid specificity remains to be revealed (26). Our lab previously found that Siglec-G negatively regulated cross-presentation in DCs through SHP1. We recently found that Src-LAPF-Cav1 promoted both bacteria and TLR4 endocytosis (37), which indicated that activating Src might increase antibacterial innate immunity. Further studies on Siglec-G in different cells by regulating SHP $1 / 2$ and Src activation may reveal the molecular mechanism of innate immune responses.

In summary, we reported that Siglec-G-induced Src signaling could orchestrate both inflammatory and anti-inflammatory cytokines by recruiting SHP1 and regulating Src activation (Supplementary Figure 5). Consistent with previous studies that revealed the negative function of Src-Syk signaling on TLR signaling, targeting Src or Siglec-G activation may be promising to treat hyper-inflammatory and hypo-inflammatory responses in sepsis.

\section{MATERIALS AND METHODS}

\section{Mice}

Mice homozygous for Siglec-G-deficient mice on a C57BL/6J background were generated as described previously (25) and bred in pathogen-free conditions. Siglecg-/- heterozygote littermates were derived from the first filial generation mice of Siglecg ${ }^{-/-}$mice mating with WT C57BL/6J mice. Six to eight weeks of age littermate mice was used in the experiments (body weight and sexuality balanced). Siglecg ${ }^{+/-}$and Siglecg ${ }^{-/-}$mice were derived from the Siglecg ${ }^{+/-}$heterozygote littermates. All animal experiments were performed in accordance with the National Institutes of Health Guide for the Care and Use of Laboratory Animals, with the approval of the Scientific Investigation Board of Second Military Medical University, Shanghai.

\section{Reagents}

LPS (O111:B4) used was described previously (11, 25). PP1 was from Calbiochem (San Diego, CA). Antibodies specific to HA-tag, myc-tag, Flag-tag [horseradish peroxidase [HRP] conjugated], CD11b (EPR1344), and the agaroses used in immunoprecipitations were from Abcam Inc. Abs specific for actin, Src, Siglec-G, p65, c-jun, HIF1 $\alpha$ and GAPDH, phospho-specific Abs against Src (Tyr416), GSK-3 $\beta$ (Ser9), STAT3 (Tyr705), p65 (Ser536), P38 (Thr180/Tyr182), ERK (Thr202/Tyr204), IKK $\alpha / \beta$ (Ser176/180), IKB $\alpha$ (Ser32), and c-jun (Ser73) were from Cell Signaling Technology. HRP-conjugated second antibody (TrueBlot) was from eBioscience. Chemical inhibitor PP1 $(5 \mu \mathrm{M})$ and zanamivir $(50 \mu \mathrm{M})$ were from Selleck company.

\section{Plasmid Constructs and Transfection}

The recombinant vectors encoding Src (NM_009271), SHP1 (NM_011202.3), HIF1 $\alpha$ (NM_001313919), STAT3 (NM_213659), and Siglec-G (NM_172900.3) were constructed by PCR-based amplification from RAW264.7 cDNA and then subcloned into the pcDNA3.1 eukaryotic expression vector (Invitrogen, San Diego, CA) as described previously $(11,25)$. The CA-Src and Siglec-G mutants were constructed as described previously $(11,25)$. Transient transfection of plasmids into HEK293T or RAW264.7 cells with jetPEI reagents (Polyplus-transfection Company) was performed following the instructions.

\section{Cell Culture and ELISA Assays}

RAW 264.7 and HEK293T cell lines were obtained from the American Type Culture Collection. Six-week-old mice were injected with $3 \%(\mathrm{w} / \mathrm{v})$ Merck thioglycollate medium into the peritoneal cavity of each mouse. Three days after the injection, the peritoneal macrophages were collected by flushing the peritoneal cavity with Roswell Park Memorial Institute (RPMI) 1640 and cultured for $1 \mathrm{~h}$ and washed with DMEM to clear the non-adherent cells; then the macrophages were further cultured in endotoxin-free DMEM with $10 \%$ fetal bovine serum (FBS) (Invitrogen) as previously reported $(11,25)$. Overexpression of Siglec-G in RAW264.7 cells was transfected with jetPEI (Polyplus) and selected by neomycin (Sigma-Aldrich) 
as described previously (11). After the stimulation (100 ng/ml of LPS), the concentrations of cytokines in the culture supernatants were determined by ELISA kits (R\&D Systems, Minneapolis, $\mathrm{MN})$ as described previously $(11,25)$.

\section{RNA Quantification}

Quantitative real-time RT-PCR analysis was performed by LightCycler (Roche, Basel, Switzerland) and SYBR RT-PCR kit (Takara, Dalian, China) as described previously $(11,25)$. Data were normalized to GAPDH expression.

\section{Immunoprecipitation and Immunoblot}

Cells were lysed with radioimmunoprecipitation assay (RIPA) buffer (Cell Signaling Technology, Beverly, MA) or M-PER Protein Extraction Reagent (Pierce, Rockford, IL) supplemented with protease inhibitor cocktail. Protein concentrations of the extracts were measured with bicinchoninic acid (BCA) assay (Pierce). The immunoprecipitation and immunoblot assays were performed as described previously $(11,25)$.

\section{Statistical Analysis}

Results are given as means plus or minus standard deviation (SD). Comparisons between two groups were performed using Student's $t$ test. Statistical significance was determined as $P$-values of $<0.05$ or 0.01 .

\section{DATA AVAILABILITY STATEMENT}

Publicly available datasets were analyzed in this study. This data can be found here: GSE39620. Other raw data supporting the conclusions of this manuscript will be made

\section{REFERENCES}

1. Bomans K, Schenz J, Sztwiertnia I, Schaack D, Weigand MA, Uhle F. Sepsis induces a long-lasting state of trained immunity in bone marrow monocytes. Front Immunol. (2018) 9:2685. doi: 10.3389/fimmu.2018.02685

2. Lin GL, McGinley JP, Drysdale SB, Pollard AJ. Epidemiology and immune pathogenesis of viral sepsis. Front Immunol. (2018) 9:2147. doi: 10.3389/fimmu.2018.02147

3. Peters van Ton M, Kox M, Abdo WF, Pickkers P. Precision immunotherapy for sepsis. Front Immunol. (2018) 9:1926. doi: 10.3389/fimmu.2018.01926

4. Sjaastad FV, Condotta SA, Kotov JA, Pape KA, Dail C, Danahy DB, et al. Polymicrobial sepsis chronic immunoparalysis is defined by diminished Agspecific T cell-dependent B cell responses. Front Immunol. (2018) 9:2532. doi: 10.3389/fimmu.2018.02532

5. Talisa VB, Yende S, Seymour CW, Angus DC. Arguing for adaptive clinical trials in sepsis. Front Immunol. (2018) 9:1502. doi: 10.3389/fimmu.2018.01502

6. Shankar-Hari M, Phillips GS, Levy ML, Seymour CW, Liu VX, Deutschman CS, et al. Developing a new definition and assessing new clinical criteria for septic shock: for the third international consensus definitions for sepsis and septic shock (Sepsis-3). JAMA. (2016) 315:775-87. doi: $10.1001 /$ jama.2016.0289

7. Singer M, Deutschman CS, Seymour CW, Shankar-Hari M, Annane D, Bauer $\mathrm{M}$, et al. The third international consensus definitions for sepsis and septic shock (Sepsis-3). JAMA. (2016) 315:801-10. doi: 10.1001/jama.2016.0287

8. Kotas ME, Medzhitov R. Homeostasis, inflammation, and disease susceptibility. Cell. (2015) 160:816-27. doi: 10.1016/j.cell.2015.02.010 available by the authors, without undue reservation, to any qualified researcher.

\section{ETHICS STATEMENT}

The animal study was reviewed and approved by Scientific Investigation Board of Second Military Medical University, Shanghai.

\section{AUTHOR CONTRIBUTIONS}

YLu, HY, and CH designed and supervised the study. WL, YLi, $\mathrm{KQ}, \mathrm{BD}$, and TL performed the experiments. WL, $\mathrm{HY}, \mathrm{CH}$, and YLu analyzed the data and wrote the paper.

\section{FUNDING}

This work was supported by grants from the National Natural Science Foundation of China (31522019, 81471568, 81871101, 81371219, 80178104, 31770945, 81541038, and 81670905) and Key Medical Talent Program of Science and Education 13th Five-Year Plan from Nantong.

\section{ACKNOWLEDGMENTS}

We thank Ms. Xiaofei Li for technical assistance.

\section{SUPPLEMENTARY MATERIAL}

The Supplementary Material for this article can be found online at: https://www.frontiersin.org/articles/10.3389/fimmu. 2019.02575/full\#supplementary-material

9. Liu J, Cao X. Cellular and molecular regulation of innate inflammatory responses. Cell Mol Immunol. (2016) 13:711-21. doi: 10.1038/cmi.2016.58

10. Cao X. Self-regulation and cross-regulation of pattern-recognition receptor signalling in health and disease. Nat Rev Immunol. (2016) 16:35-50. doi: $10.1038 /$ nri.2015.8

11. Han C, Jin J, Xu S, Liu H, Li N, Cao X. Integrin CD11b negatively regulates TLR-triggered inflammatory responses by activating Syk and promoting degradation of MyD88 and TRIF via Cbl-b. Nat Immunol. (2010) 11:734-42. doi: 10.1038/ni.1908

12. Ivashkiv LB. How ITAMs inhibit signaling. Sci Signal. (2011) 4:pe20. doi: 10.1126/scisignal.2001917

13. Abram CL, Lowell CA. The expanding role for ITAM-based signaling pathways in immune cells. Sci STKE. (2007) 2007:re2. doi: 10.1126/stke.3772007re2

14. Hu $\mathrm{X}$, Han $\mathrm{C}$, Jin J, Qin $\mathrm{K}$, Zhang $\mathrm{H}$, Li $\mathrm{T}$, et al. Integrin CD11b attenuates colitis by strengthening Src-Akt pathway to polarize antiinflammatory IL-10 expression. Sci Rep. (2016) 6:26252. doi: 10.1038/srep 26252

15. Goncalves-de-Albuquerque CF, Rohwedder I, Silva AR, Ferreira AS, Kurz A. R., M.,Cougoule C, et al. The Yin and Yang of tyrosine kinase inhibition during experimental polymicrobial sepsis. Front Immunol. (2018) 9:901. doi: 10.3389/fimmu.2018.00901

16. Fraschilla I, Pillai S. Viewing Siglecs through the lens of tumor immunology. Immunol Rev. (2017) 276:178-91. doi: 10.1111/imr.12526

17. Mahajan VS, Pillai S. Sialic acids and autoimmune disease. Immunol Rev. (2016) 269:145-61. doi: 10.1111/imr.12344 
18. Pillai S, Netravali IA, Cariappa A, Mattoo H. Siglecs and immune regulation. Annu Rev Immunol. (2012) 30:357-92. doi: 10.1146/annurev-immunol-020711-075018

19. Crocker PR, Paulson JC, Varki A. Siglecs and their roles in the immune system. Nat Rev Immunol. (2007) 7:255-66. doi: 10.1038/nri2056

20. Wang J, Sun J, Liu LN, Flies DB, Nie X, Toki M, et al. Siglec-15 as an immune suppressor and potential target for normalization cancer immunotherapy. Nat Med. (2019) 25:656-66. doi: 10.1038/s41591-019-0374-x

21. Li N, Zhang W, Wan T, Zhang J, Chen T, Yu Y, et al. Cloning and characterization of Siglec-10, a novel sialic acid binding member of the Ig superfamily, from human dendritic cells. J Biol Chem. (2001) 276:28106-12. doi: 10.1074/jbc.M100467200

22. Ding Y, Guo Z, Liu Y, Li X, Zhang Q, Xu X, et al. The lectin Siglec-G inhibits dendritic cell cross-presentation by impairing MHC class I-peptide complex formation. Nat Immunol. (2016) 17:1167-75. doi: 10.1038/ni.3535

23. Chen GY, Chen X, King S, Cavassani KA, Cheng J, Zheng X, et al. Amelioration of sepsis by inhibiting sialidase-mediated disruption of the CD24-SiglecG interaction. Nat Biotechnol. (2011) 29:428-35. doi: $10.1038 /$ nbt.1846

24. Chen GY, Tang J, Zheng P, Liu Y. CD24 and Siglec-10 selectively repress tissue damage-induced immune responses. Science. (2009) 323:1722-5. doi: $10.1126 /$ science. 1168988

25. Chen W, Han C, Xie B, Hu X, Yu Q, Shi L, et al. Induction of Siglec-G by RNA viruses inhibits the innate immune response by promoting RIG-I degradation. Cell. (2013) 152:467-78. doi: 10.1016/j.cell.2013.01.011

26. Bordon Y. Inflammation: Live long and prosper with Siglecs. Nat Rev Immunol. (2015) 15:266-7. doi: 10.1038/nri3851

27. Gruber S, Hendrikx T, Tsiantoulas D, Ozsvar-Kozma M, Goderle L, Mallat Z, et al. Sialic acid-binding immunoglobulin-like lectin $\mathrm{G}$ promotes atherosclerosis and liver inflammation by suppressing the protective functions of B-1 cells. Cell Rep. (2016) 14:2348-61. doi: 10.1016/j.celrep.2016.02.027

28. Ivashkiv LB. A signal-switch hypothesis for cross-regulation of cytokine and TLR signalling pathways. Nat Rev Immunol. (2008) 8:816-22. doi: $10.1038 /$ nri2396

29. Qin K, Han C, Zhang H, Li T, Li N, Cao X. NAD(+) dependent deacetylase Sirtuin 5 rescues the innate inflammatory response of endotoxin tolerant macrophages by promoting acetylation of p65. J Autoimmun. (2017) 81:120-9. doi: 10.1016/j.jaut.2017.04.006

30. Ouyang W, Rutz S, Crellin NK, Valdez PA, Hymowitz SG. Regulation and functions of the IL-10 family of cytokines in inflammation and disease. Annu Rev Immunol. (2011) 29:71-109. doi: 10.1146/annurev-immunol-031210-101312

31. An H, Hou J, Zhou J, Zhao W, Xu H, Zheng Y, et al. Phosphatase SHP1 promotes TLR- and RIG-I-activated production of type I interferon by inhibiting the kinase IRAK1. Nat Immunol. (2008) 9:542-50. doi: 10.1038/ni.1604

32. Gilroy DW, Yona S. HIF1alpha allows monocytes to take a breather during sepsis. Immunity. (2015) 42:397-9. doi: 10.1016/j.immuni.2015.02.016

33. Zhu J, Luo L, Tian L, Yin S, Ma X, Cheng S, et al. Aryl hydrocarbon receptor promotes IL-10 expression in inflammatory macrophages through Src-STAT3 signaling pathway. Front Immunol. (2018) 9:2033. doi: $10.3389 /$ fimmu.2018.02033

34. Horiguchi H, Loftus TJ, Hawkins RB, Raymond SL, Stortz JA, Hollen MK, et al. Innate immunity in the persistent inflammation, immunosuppression, and catabolism syndrome and its implications for therapy. Front Immunol. (2018) 9:595. doi: 10.3389/fimmu.20 18.00595

35. Muller J, Lunz B, Schwab I, Acs A, Nimmerjahn F, Daniel C, et al. Siglec-G deficiency leads to autoimmunity in aging C57BL/6 Mice. J Immunol. (2015) 195:51-60. doi: 10.4049/jimmunol.1403139

36. Barkal AA, Brewer RE, Markovic M, Kowarsky M, Barkal SA, Zaro BW, et al. CD24 signalling through macrophage Siglec-10 is a target for cancer immunotherapy. Nature. (2019) 572:392-6. doi: 10.1038/s41586-0191456-0

37. Li T, Qin K, Li N, Han C, Cao X. An endosomal LAPF is required for macrophage endocytosis and elimination of bacteria. Proc Natl Acad Sci USA. (2019) 116:12958-63. doi: 10.1073/pnas.19038 96116

Conflict of Interest: The authors declare that the research was conducted in the absence of any commercial or financial relationships that could be construed as a potential conflict of interest.

Copyright (c) $2019 \mathrm{Li}, \mathrm{Li}$, Qin, Du, Li, Yuan, Han and Luo. This is an open-access article distributed under the terms of the Creative Commons Attribution License (CC $B Y)$. The use, distribution or reproduction in other forums is permitted, provided the original author(s) and the copyright owner(s) are credited and that the original publication in this journal is cited, in accordance with accepted academic practice. No use, distribution or reproduction is permitted which does not comply with these terms. 